\title{
Die Flucht vor Gewalt? Stereotype und Motivationen beim Andrang auf barrios privados in Buenos Aires
}

\section{Michael Janoschka, Berlin ${ }^{1)}$}

\section{Einleitung: Ein Zusammenhang zwischen Kriminalität und barrios privados?}

«In Argentinien wohnen immer mehr Menschen aus Angst vor Überfällen in geschlossenen Siedlungen» titelte die Frankfurter Allgemeine Zeitung erst vor kurzem (OeHrLein 2002) und reihte sich damit in die Berichterstattung über bewachte Wohnkomplexe in deutschsprachigen Massenmedien ein, die immer wieder die Bedrohung durch Kriminalität als zentrale Motivation für den Umzug in eine bewachte Wohnanlage herausstreichen (vgl. SiEgELE 1996; RADA 1999). Aber auch die sozialwissenschaftliche Analyse privater Stadtviertel geht von diesem kausalen Zusammenhang oft ohne empirischen Nachweis quasi als «Naturgesetz» aus. Diese Annahme entbehrt einer unvoreingenommenen wissenschaftlichen Position und postuliert die Trennung der Städte in Trutzburgen der Autosegregation und hoch gefährliche Räume (DAvIS 1990; BORJa \& CASTElls 1997; Soja 2000). In Untersuchungen nordamerikanischer gated communities (vgl. BLAKely \& SNyder 1997; Wehrheim 1999; Amendola 2000) wird die Wanderung der Wohlhabenden und der Mittelschicht in diese Wohnform ebenso in erster Linie mit dem zunehmenden Sicherheitsbedürfnis erklärt wie bei den Fallstudien zu Lateinamerika (vgl. Caldeira 2000; Coy 2001; Hiernaux-Nicolás 1999; Marti I Puig 2001; Prévôt-Schapira 2000; Dammert 2001). Für einige Städte wie Mexiko-Stadt oder São Paulo mag die vorgestellte These empirische Gültigkeit besitzen, in vielen anderen Fällen werden durch diese Annahmen Vereinfachungen und Verzerrungen induziert. Dies unterstreichen kritische Betrachtungen über das Phänomen bewachter Wohnkomplexe in Nordund Südamerika. Trotz der hohen Kriminalitätsrate in den USA (vgl. Lichtenberger 1998: 334) begründet Massey (1999) in Anlehnung an Sennetr (1971), die Umzäunung der suburbanen Wohngebiete stehe in direkter Verbindung zur Suche nach einem sozial homogenen Wohnumfeld. Ebenso werden die wirtschaftsliberale Steuerung von Stadtentwicklung, das damit in Zusammenhang stehende Steuerrecht und die restriktiven, auf den Ausschluss von ökonomisch schwächeren Haushalten ausgerichteten Flächennutzungspläne der Umlandgemeinden als bedeutsame Faktoren für die massive Entstehung der umzäunten Wohnviertel aufgeführt (GLasze 2002; Müller \& ROHR-ZänKer 2001). Low (2002) zeigt, dass im texanischen San Antonio trotz der zweitniedrigsten Kriminalitätsrate in allen US-Großstädten der Markt neugebauter Einfamilienhäuser fast nur noch aus gated communities besteht. Auch in Lateinamerika muss über den Sicherheitsaspekt hinausgehend die Vernachlässigung der öffentlichen Infrastruktur durch die staatliche Verwaltung berücksichtigt werden ( $\mathrm{vgl}$. BäHr \& Mertins 1995). Der Rückzug des Staates aus sozialer Umverteilung und Stadtentwicklung im Zuge der neoliberalen Politikgestaltung hat die staatliche Interventionsbasis erodiert. Darüber hinaus ist das Vertrauen in den Staat aufgrund von Korruption und Vetternwirtschaft weitgehend diskreditiert (vgl. JANOSCHKA 2002b).

Die Darstellung und Interpretation der persönlichen Handlungsmuster und Beweggründe der Bewohner von barrios privados im Metropolenraum von Buenos Aires, die auf einer Analyse von Lebensstilen und persönlichen Biographien basiert, soll nachfolgend eine differenzierte Herangehensweise an die Thematik eröffnen und Unterschiede zur Argumentation im Beitrag über Quito aufzeigen. Dem monokausalen Zusammenhang zwischen Kriminalität und physischer Sicherung des Wohnumfeldes wird widersprochen. Trotz eines latenten, aber in erster Linie medial zugespitzten Diskurses wachsender Kriminalität und Brutalität in Buenos Aires und der Verschärfung sozialer Kontraste innerhalb der argentinischen Gesellschaft stellen diese Aspekte nur einen untergeordneten Gesichtspunkt bei der Entscheidung dar, den Lebensmittelpunkt in privat geschützte Wohngebiete zu verlegen.

\section{Stadträumliche Veränderungen: Die Typisierung der bewachten Wohnkomplexe in Buenos Aires}

Die zehnjährige Phase (1991-2001) neoliberaler Wirtschaftspolitik, die von der Privatisierung von Staatsbetrieben, der Anbindung des argentinischen Pesos an den US-Dollar und der Zunahme offener Arbeitslosigkeit trotz hohen Wirtschaftswachstums geprägt war,

\footnotetext{
1) Für die Gesamterhebung siehe JanOSCHKA 2002b, ISR-Forschungsbericht 28, Verlag der Österreichischen Akademie der Wissenschaften (im Druck).
} 


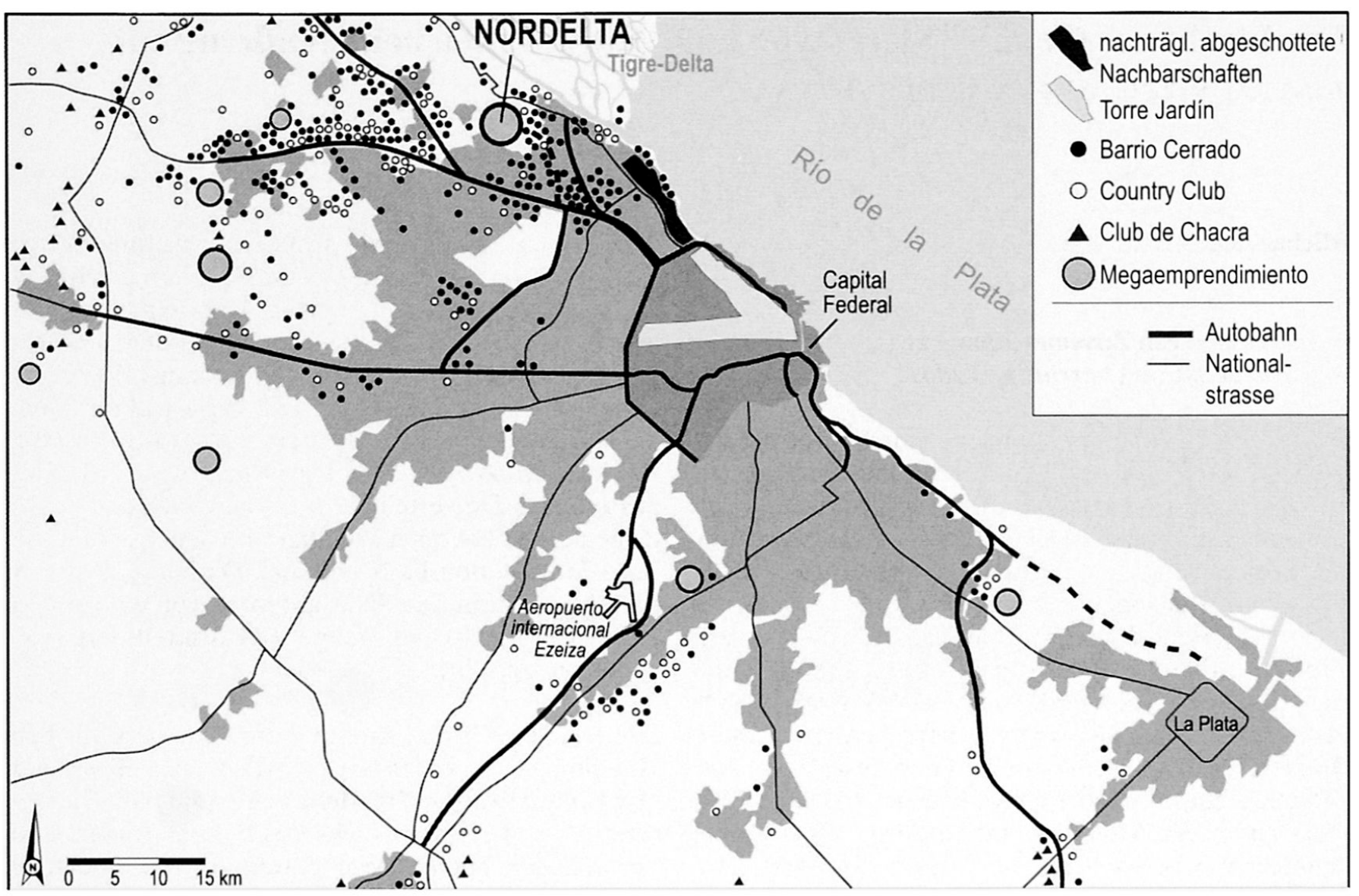

Abb. 1: Die räumliche Verteilung bewachter Wohnkomplexe im Grossraum Buenos Aires

The spatial distribution of gated communities in Greater Buenos Aires

La distribution spatiale des quartiers enclos dans le Grand Buenos Aires

Kartengrundlage: Tageszeitung LA NACIÓN, 10.11.2001; JANOSCHKA 2002b; Kartographie: M. JANOSCHKA

hat sich die Schere zwischen Arm und Reich weiter denn je geöffnet. Schon vor dem Ausbruch der akuten Wirtschafts- und Finanzkrise im Herbst 2001 verzeichneten in der Stadtregion Buenos Aires 80 Prozent aller Bewohner reale Einkommensverluste, während nur das obere Quintil hinzugewann (WelCh Guerra 2002: 74). Ein Teil dieser 20 Prozent hat in diesem Zeitraum die Metropole in erheblichem Maße verändert. Massive Investitionen in das zu Beginn der neunziger Jahre an private Konsortien konzessionierte Autobahnnetz legten die Grundlage dafür, dass der suburbane Raum für die mittlere Mittelschicht und Teile der oberen Mittelschicht zu einer attraktiven und gut an das Stadt- und Bürozentrum angebundenen Wohnalternative wurde (Blanco 1999; YANES et.al. 1996; GILBERT 1998). Seit Mitte der neunziger Jahre entstand im suburbanen Raum ein wahrer Boom von barrios privados mit mehr als 450 Anlagen für eine halbe Million Bewohner. Gleichzeitig entwickelte sich begleitend auch die notwendige Infrastruktur inmitten der bis dahin durch niedrige Einkommensschichten geprägten Siedlungslandschaft des suburbanen Umlands von Buenos Aires: Einkaufszentren, Privatschulen, Universitäten, Filialen teurer Restaurants aus dem Stadtzentrum und Urban Entertainment Center sind inzwischen in der gesamten Stadtregion verstreut (CICCOLELla 1998; JaNOSCHKa 2000; JANOSChKa 2002c). Darüber hinaus wird auch der Umbau bestehender Stadtviertel in zentralen Bereichen vorangetrieben.

Beide Entwicklungen führen zu einer direkten Nachbarschaft von Gegensätzlichem: Reichtum trifft auf extreme Armut und Verfall, und wohlhabende Schichten annektieren traditionelle Aktionsräume der Unterschicht am Rand der Städte. Die geschützten privaten Wohngebiete lassen sich in sechs Kategorien einteilen (vgl. JANOSCHKA 2002a; Abb. 1):

- Die nachträglich abgeschottete Nachbarschaft: In vereinzelten Fällen wurde der Zugang zu Einfamilienhausgebieten im Bestand zugangsbeschränkt (Foto 1). Wesentlich weiter verbreitet ist dagegen 


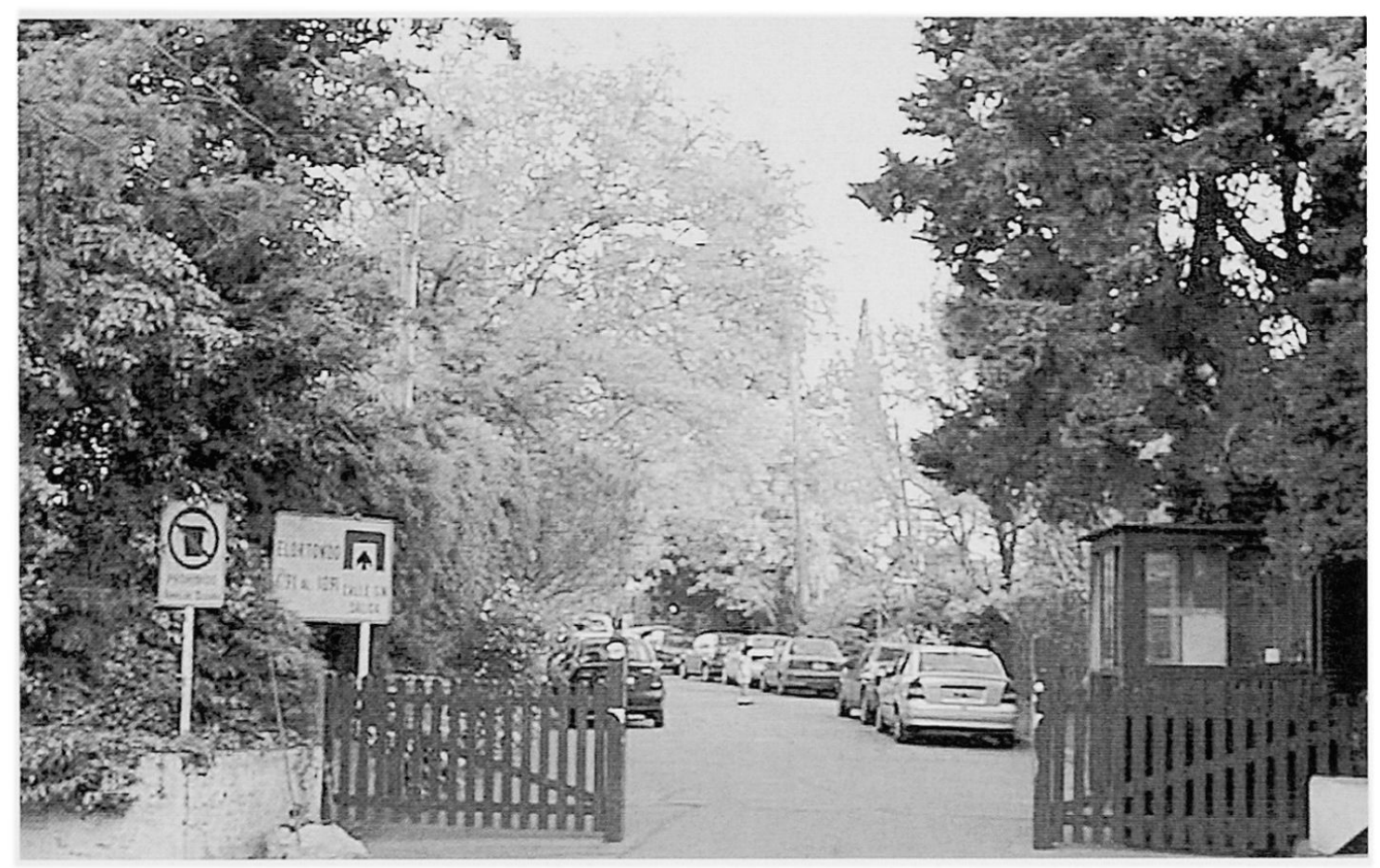

Foto 1: Immer mehr Wohngebiete der Oberschicht werden - oftmals illegal - nachträglich abgesperrt. More and more neighbourhoods of the upper classes are enclosend - in most cases illegally.

Un nombre croissant d'aires résidentielles sont encloses - souvent illégalement - au bénéfice des couches supérieures de la population.

Foto: M. JANOSCHKA

die Überwachung des Straßenraums durch private Wachdienste.

- Das zentrumsnahe condominio vertical (Torre Jardín): Darunter werden Hochhäuser verstanden, welche mehrere Meter zurückgezogen von der Straßenfront errichtet und durch Zäune und Sicherheitspersonal vom öffentlichen Raum getrennt werden. Sportanlagen und eigene Grünflächen für die Bewohner sind Bestandteil dieses Konzepts.

- Das suburbane barrio cerrado. Dabei handelt es sich um umzäunte und zugangsbeschränkte Neubauviertel der Mittelschicht am Standrand, in der Regel Einfamilienhaussiedlungen auf vergleichsweise kleinen Parzellen.

- Der suburbane Country Club. Fußballplätze, Tennis-, Golf-, Polo- oder Reitanlagen sind ein wesentlicher Bestandteil der Gebiete, von denen ursprünglich manche als Wochenendsiedlungen gegründet wurden. Inzwischen hat sich die Mehrzahl der Clubs aber in Erstwohnsitze verwandelt. Das Publikum ist wesentlich finanzkräftiger als in den barrios cerrados (Foto 2).
- Der semirurale Club de Chacra: Chacras sind traditionelle Landhäuser der Mittel- bis Oberschicht auf normalerweise mehreren Hektar großen Grundstücken, die vor allem in den dreimonatigen Schulferien im Sommer bewohnt werden. Inzwischen sind auch bewachte und umzäunte Clubs dieses Typs errichtet worden.

- Das suburbane Megaemprendimiento. Mit dem Verkaufsstart des Projekts Nordelta im Jahr 1999 ist eine neue Kategorie entstanden, die vorherige Größenordnungen sprengt. Bei den inzwischen neun Megaemprendimientos handelt es sich um Bauvorhaben mit mehreren tausend Wohneinheiten. Neben Sport- und Freizeitanlagen sind eigene Privatschulen und zusätzliche Infrastruktur bis hin zu Einkaufszentren vorgesehen. Gleichzeitig werden preislich und räumlich differenzierte Hausund Grundstücksgrößen sowie teilweise auch Apartmentwohnungen angeboten. Das Nordelta, das als ciudadpueblo (= Stadtdorf) für etwa 80.000 Einwohner vermarktet wird, sprengt sogar diese Größenordnung. Es bietet auch eine Berufsschule, eine Privatuniversität, kulturelle Einrichtungen und soll auch Büroarbeitsplätze erhalten. Damit entsteht erstmals eine "private Stadt» im Umland 


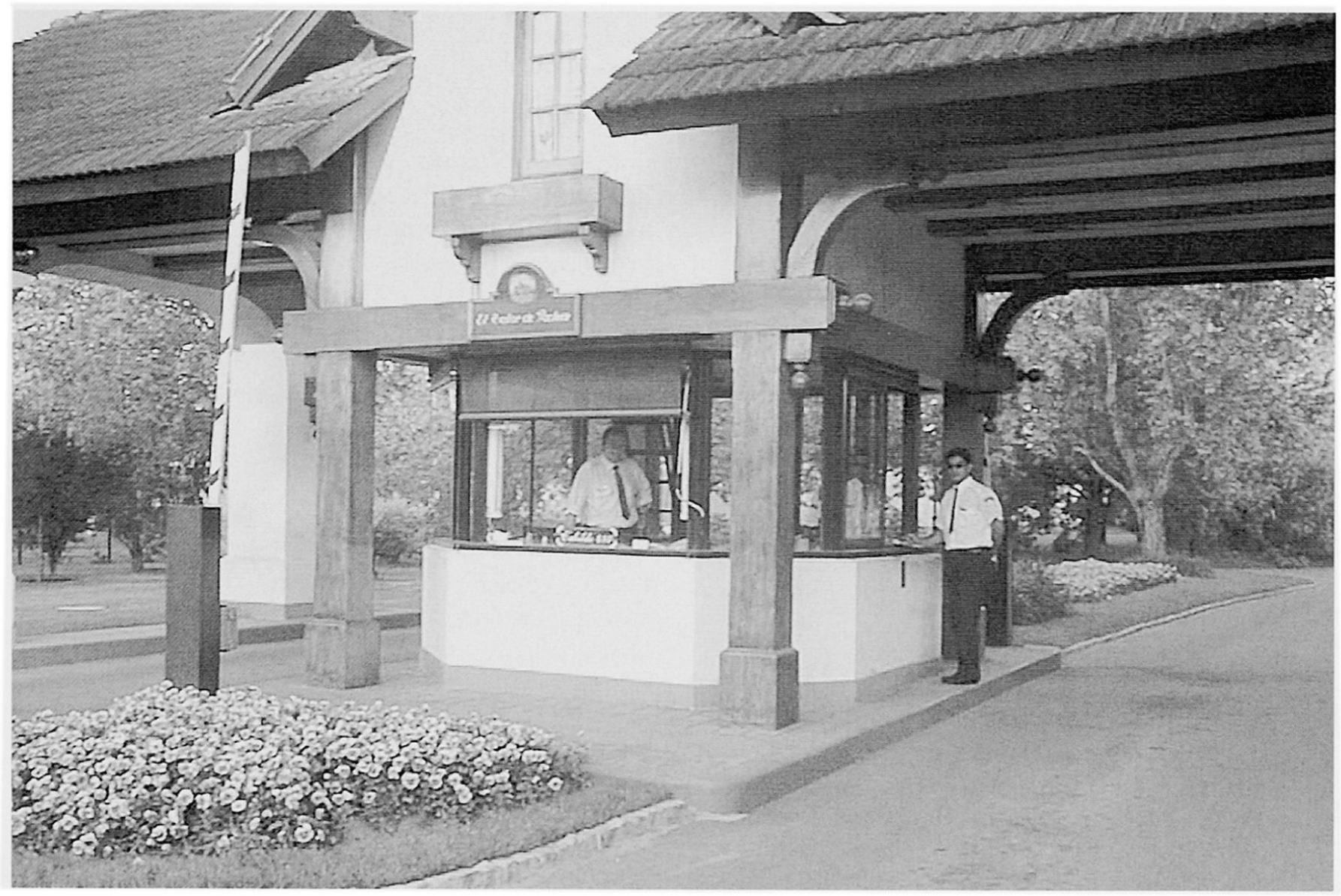

Foto 2: Repräsentative Einfahrten charakterisieren den Status von Country Clubs der oberen Mittelschicht. Representative entrance gates characterize the status of upper middle class Country Clubs.

Des entrées représentatives caractérisent le statut du Country Club de la bourgeoisie moyenne.

Foto: M. JANOSCHKA

von Buenos Aires, die durch einen eigenen Autobahn- und Bahnanschluss mit dem Stadtzentrum verbunden wird. Wie in einer gewachsenen Stadt gibt es unterschiedliche Funktions- und Nutzungszonen und unterschiedliche Dichtewerte und Bodenpreise (vgl. JANOSCHKA 2002b).

\section{Methodisches Untersuchungsdesign}

Systematisch durchgeführte Bewohnerinterviews erfolgten in den privaten Nachbarschaften der lateinamerikanischen Großstädte bislang nur in Einzelfällen. Da die Fragestellung vor allem auf die Untersuchung handlungserklärender und -begründender Motive für das raumprägende Umzugsverhalten der neuen Bewohner privater Wohnviertel abzielt und bislang nur wenig erforschte Wirklichkeitsbereiche untersucht wurden, erscheinen Methoden der qualitativen Sozialforschung angepasster (vgl. FLICK; v. KARDORFF \& STEINKE 2000). Die nachfolgenden Ergebnisse aus Befragungen, die zwischen November 2001 und Januar
2002 durchgeführt wurden, basieren auf der Anwendung von zwei methodischen Verfahren:

1. Biographisch-narrative Interviews mit 15 Bewohnern und Grundstückseigentümern des Nordelta, die nach SchüTZE in vier Schritten ablaufen: Einer Erzählaufforderung folgt die autobiographische Anfangserzählung, die vom interviewenden Forscher möglichst nicht unterbrochen wird. Erst nach Beendigung dieser Haupterzählung (durch ein klares Signal, z.B. «so, das war's») werden Nachfragen gestellt, um das tangentielle Erzählpotenzial abzuschöpfen. Die ergänzenden Fragen beziehen sich fallspezifisch auf die vorherigen Aussagen und sollen den Interviewpartner erneut zu weiterführenden, vertiefenden Erzählungen anregen. Erst im letzten Teil des Interviews können dann auch vertiefende Fragen gestellt werden, welche bis dahin unberührte Themen aufgreifen und für den Forscher von Interesse sind (SCHÜTZE 1983; Fischer-Rosenthal \& Rosenthal 1997). 
2. Gruppendiskussion mit den Müttern einer der Privatschulen im Nordelta, welche aufgrund ihrer Nähe zu normalen, alltäglichen Diskussionen schnell den Abbau von Gesprächshemmungen zeigt: Indirekte, nur im Gruppenkontext geäußerte Meinungsbilder und Verhaltensweisen kommen auf diesem Wege zum Vorschein (Loos \& SCHÄFFER 2001; LAMNEK 1998). Darüber hinaus sind tiefere und detailliertere Meinungen abfragbar. Mittels eines provokanten Grundreizes eingeleitet und weitgehend ungesteuert greift der Moderator lediglich bei Gesprächspausen mit neuen Thesen, Rückfragen oder Reizen ein (vgl. Lamnek 1993). Die interviewte Gruppe setzte sich aus Elternteilen einer Privatschule zusammen, die sowohl innerhalb der privaten Stadt als auch in anderen privaten Wohngebieten leben.

Ferner fliessen in die Betrachtungen Erkenntnisse aus 40 Experteninterviews in Buenos Aires ein. Die Anwendung einer solchen methodischen Triangulation erfolgt unter der Prämisse, dass so die Validität der Ergebnisse maximiert wird (Denzin 1978; Flick 2000). Aufschlussreich ist diese Triangulation daher, weil sie unterschiedliche Perspektiven verdeutlichen kann und damit eine tiefere und besser passende Theorieentwicklung und Abstraktion ermöglicht (GLASER \& Strauss 1998). Das gesammelte Datenmaterial, aufgenommen und transkribiert, wurde nachfolgend anhand des Verfahrens der qualitativen Inhaltsanalyse nach MAYRING bearbeitet (vgl. MAYRING 1991, 1999, 2000).

\section{Umzugsmotivationen und Biographietypen von Bewohnern im Nordelta}

Das Nordelta als das Beispiel der größten der im Bau befindlichen privaten Städte und dörflichen Siedlungen im Umland von Buenos Aires ist ein Zufluchtsort für die vom Chaos, der Hektik und den schlechten Lebensverhältnissen der argentinischen Hauptstadt gestressten Bewohner. Konzeption und Vermarktung stehen im Zeichen der Annehmlichkeiten, die bei nur 30 Minuten Fahrzeit ins Zentrum die Nähe zu Buenos Aires mit ländlich-naturnahen Lebensformen kombinieren. Im Nordelta wird es Kinos, Schulen und Einkaufszentren in direkter Umgebung geben, ohne dass die Bewohner aber die schlechte Luft, die Enge, den Lärm und auch die Armut der Großstadt ertragen müssen. Ähnliche Marketingkonzepte verfolgen auch die kleineren Megaemprendimientos wie z.B. Pilar del Este: In direkter Nachbarschaft des privaten Wohngebiets für zukünftig etwa 12.000 Menschen baute der Investor einen öffentlich zugänglichen Dorfkern, der aber nur über eine bewachte Privatstraße ohne Anschluss an das öffentliche Transportnetz erreichbar ist. Dort finden nicht nur Gottesdienste und Freizeitveranstaltungen statt, sondern inzwischen werden auch traditionelle Stadtfeste an diesen semiprivaten Ort verlagert.

Die Motivationen der interviewten Bewohner für ihren Umzug in die private Stadt Nordelta bedecken unterschiedliche Ebenen: Einerseits ist das Set stadträumlicher Veränderungen zu nennen, das mit den politischen und ökonomischen Transformationen der 1990er Jahre eher als Makro-Kraft im Hintergrund wirkt. Der Verfall eines staatlichen Gemeinwesens führt zu der Suche nach einer privatwirtschaftlich organisierten und persönliche Vorteile versprechenden Wohnlösung. Dabei kommen alltägliche Aktionsmuster, die vielfach von zunehmender Rücksichtslosigkeit und Egoismus geprägt sind, auch bei der Wohnortwahl zur Anwendung. Soziale Homogenität spielt eine wichtige Rolle: Nachbarn in der gleichen Lebensphase und aus gleichen ökonomischen Verhältnissen steigern die innere Kohärenz des Wohnviertels und lassen ein Gemeinschaftsgefühl erwachen. An diesem Punkt erfolgt die Zusammenführung von Makro-Faktoren und den persönlichen Beweggründen, die in direktem Zusammenhang mit der persönlichen Biographie stehen: Käufer und Bauherren im Nordelta besitzen eine außerordentlich hohe Homogenität. Der überwiegende Teil sind junge Familien im Alter von 35 bis 40 Jahren mit zwei Kindern, die gerade schulpflichtig werden oder im Kindergartenalter sind. Zumindest ein Elternteil hat die Hochschulausbildung abgeschlossen, in der Regel beide.

In vorderster Linie steht bei den Eltern der markante Wunsch nach einer Veränderung im Lebensstil, der aus einem Überdruss der schlechten Lebensbedingungen in zentralen Stadtbereichen resultiert. Wohnen im Grünen und Bewegungsfreiheit für die Kinder sind die zentralen Argumente der jungen Familien. Erinnerungen an Kindheits- und Jugenderfahrungen spielen bei der Wahl des neuen Wohnorts eine entscheidende Rolle. Darüber hinaus spielen auch die Erfahrungen von Freunden und Verwandten, die in der zweiten Hälfte der 1990er Jahre in andere private Wohnviertel gezogen sind, eine entscheidende Rolle.

Es lassen sich vier biographische Typen (vgl. Tab. 1) deuten, die unterschiedliche räumliche Erfahrungshorizonte und Sozialisationsmuster besitzen. Sämtliche Personen- bzw. Typenbezeichnungen werden geschlechtsneutral verwandt:

1. Der porteño: Geboren und aufgewachsen in der Capital Federal (Capital Federal ist der innerstädtische und politisch unabhängige Teil der Agglomeration Buenos Aires, vgl. Abb. 1. In der Autodefinition der Bürger ist diese sich auch im ökonomischen Bereich manifestierende Trennung «Capital» und «Provincia» ständig präsent.) hat diese Per- 


\begin{tabular}{|l|l|l|l|l|l|l|}
\hline & Geburtsort & Sozialisationsort & Wohnform & $\begin{array}{l}\text { Erfahrung } \\
\text { Grün }\end{array}$ & $\begin{array}{l}\text { Ökonom. } \\
\text { Profil* }\end{array}$ & Arbeitsort \\
\hline porteño & $\begin{array}{l}\text { Capital } \\
\text { Federal }\end{array}$ & $\begin{array}{l}\text { Capital Federal } \\
\text { Ausland }\end{array}$ & Appartement & $\begin{array}{l}\text { Campo, Quin- } \\
\text { ta, Chacra }\end{array}$ & A, B & $\begin{array}{l}\text { Capital Federal } \\
\text { homework }\end{array}$ \\
\hline bonaerense & $\begin{array}{l}\text { Buenos Aires: } \\
\text { Zona Norte }\end{array}$ & $\begin{array}{l}\text { Buenos Aires } \\
\text { Ausland }\end{array}$ & Einfamilienhaus & $\begin{array}{l}\text { Quinta } \\
\text { Country } \\
\text { Club }\end{array}$ & A, B & $\begin{array}{l}\text { Capital Federal, } \\
\text { Zona Norte }\end{array}$ \\
\hline $\begin{array}{l}\text { porteño } \\
\text { suburbano }\end{array}$ & $\begin{array}{l}\text { Capital } \\
\text { Federal }\end{array}$ & Capital Federal & Appartement & - & C 1 & Suburban \\
\hline $\begin{array}{l}\text { proveniente } \\
\text { del interior }\end{array}$ & Landesinnere & $\begin{array}{l}\text { Landesinnere } \\
\text { Capital Federal }\end{array}$ & Appartement & $\begin{array}{l}\text { Kleinstadt } \\
\text { Dorf }\end{array}$ & C 1 & Capital Federal \\
\hline
\end{tabular}

* Immobilienmakler unterscheiden die Klassen A (Oberschicht), B (obere Mittelschicht) und C 1. Die Belegung dieser Klassen mit konkreten Einkommensziffern ist sehr schwierig, die Immobilienfirmen definieren die Klassen unterschiedlich und nach der Abkopplung des argentinischen Pesos vom US-Dollar sind genaue Klassifizierungen momentan hinfällig. Vor der Abwertung bedeutete C 1 ungefähr $1.800-$ 3.000 US\$ Haushaltseinkommen pro Monat, B etwa $3.000-6.000$ US\$, A > 6.000 US\$. C 1 ist in der Regel die niedrigste Einkommensschicht, die von den Immobilienfirmen als Zielgruppe für private Wohnkomplexe in Betracht kommt.

Tab. 1: Biographische Typen der Bewohner des Nordelta Biographic types of "Nordelta" inhabitants

Les types biographiques des habitants du "Nordelta"

sonengruppe ihr bisheriges Leben in den zentralen Stadtbereichen organisiert. Die Familien haben, da auch die Eltern aus der Capital Federal stammen, fast ausschließliche Lebenserfahrungen in mehrstöckigen Wohnhäusern. Der Wunsch nach dem Haus im Grünen ergibt sich aus den Kindheitserfahrungen, als die dreimonatigen Schulferien im Sommer vollständig im Großfamilienkreis auf einem Landbesitz außerhalb von Buenos Aires verbracht wurden (Campo, Quinta, Chacra genannt, je nach Lage und Grösse). Darüber hinaus besteht der Wunsch, den Kindern eine Nachbarschaft zu bieten, in der sie sich frei, mit ausreichend Grünflächen und ohne Angst vor Autounfällen entfalten können. Der porteño verfügt über Auslandserfahrung (USA oder Europa) im Rahmen des Studiums, durch enge Verwandte oder zeitweilige Arbeitsaufenthalte durch Anstellungen in multinationalen Konzernen.

2. Der bonaerense: Geboren und aufgewachsen in den nördlich an die Capital Federal angrenzenden Gemeinden sind die räumlichen Organisationsmuster des Lebens dieser Gruppe wesentlich differenzierter: Über die direkte Nachbarschaft hinausgehend unterhalten diese Bewohner auch enge Verbindungen in die Capital Federal, oft aufgrund beruflicher Verpflichtungen. Parallel dazu verläuft das Bestreben, möglichst viele berufliche Aktivitäten im eigenen Haus zu verrichten. Der bonaerense ist in Einfamilienhäusern aufgewachsen, die allerdings keine Gärten besitzen. Direkte Erfahrungen mit Grünflächen stammen entweder aus den Schulferien oder aus dem (zeitweiligen) Besitz eines Wochenendhauses durch die Eltern. Auch der bonaerense besitzt Auslandserfahrung, beide Typen haben ein vergleichbar hohes ökonomisches Profil (obere Mittelschicht).

3. Der porteño suburbano: Geboren in der Capital Federal besitzt dieser Typus ausschließliche Lebenserfahrungen in mehrstöckigen Wohnhäusern. Die Umzugsmotivation richtet sich weniger nach dem Lebensstil im Grünen, sondern ist pragmatisch ausgerichtet, da der Hauptverdiener im suburbanen Raum der Metropole arbeitet. Das Herausziehen aus dem Stadtzentrum in die private Stadt ist damit mit erheblicher Zeit- und Kostenersparnis verbunden. Dieser Faktor spielt für die deutlich niedrigeren finanziellen Spielraum besitzenden Familien dieses Typus eine wichtige Rolle.

4. Der proveniente del interior: Geburt oder lang- 
jährige Aufenthalte in den Provinzen im Landesinnern, in Kleinstädten oder im ländlichen Raum kennzeichnen diesen Typus. Daher stammt die Erfahrung des intensiven Kontakts mit der Natur. Nach dem Umzug nach Buenos Aires wird die fehlende Natur vermisst und als wesentlicher Beweggrund für den Umzug aufgeführt. Das sozioökonomische Niveau entspricht der Mittelschicht.

Diese vier Typen prägen in ihrer Argumentation einen Diskurs, der sich vor allem um die Aspekte «Leben im Grünen», «ruhiges und friedliches Wohnumfeld», «bessere Konditionen für die Kinder» und «frische Luft» dreht. Die spezielle Entscheidung für das Nordelta hängt zudem damit zusammen, dass der national für seine erfolgreichen Finanzgeschäfte bekannte Unternehmer und Börsenhändler EdUARDo CONSTANTINI mit seinem vertrauenswürdigen Namen für das gigantische Bauprojekt bürgt. Außerdem denken viele Eltern an die Zukunft und ihre eigenen Erfahrungen, der neue Wohnort soll den Kindern auch in der Phase des Erwachsenwerdens und des Studiums ein Zuhause bleiben können, die Unabhängigkeit vom Stadtzentrum ist mit den Infrastruktureinrichtungen gewährleistet.

Die persönlichen Erfahrungen der Pioniere des Nordeltas stehen in Gegensatz zu dem hysterisch überzogenen medial-öffentlichen Diskurs in der argentinischen Gesellschaft. Wichtige Funktionäre staatlicher und privater Institutionen im Bereich der Stadt- und Regionalentwicklung vertreten in persönlichen Gesprächen eine Auffassung, welche sich mit der Darstellung in den großen nationalen Zeitungen deckt und Buenos Aires als extrem gefährlich und unsicher erscheinen lässt. Jede einzelne Gewalttat wird medial ausgeschlachtet und bleibt so wochenlang im Gedächtnis der Bewohner haften. Tatsächlich ist Buenos Aires - trotz des starken Anstiegs an Verbrechen im letzten Vierteljahrhundert - noch immer eine vergleichsweise sichere Stadt, in der selbst der ausländische Besucher in den meisten Stadtvierteln nachts ohne große Bedrohung flanieren kann.

Der mediale Eindruck wird durch die Vermarktungsstrategien der Immobilienfirmen gestützt. In Experteninterviews mit den im Nordelta tätigen Immobilienmaklern wurden die Sicherheitsaspekte als wesentliche Säulen der Vermarktung im Stil des Gewaltdiskurses präsentiert. Den interviewten Bewohnern hingegen ist diese Argumentation weitgehend fremd. Die Bewachung der Siedlung ist Teil eines Pakets, das gekauft wird und zu dem es auf dem Immobilienmarkt keine Alternative gibt. Suburbanes Wohnen ist für die Mittel- und obere Mittelschicht im Grunde nur in dieser Form möglich und wird durch eine Koalition aus Immobilienfirmen und den großen Tageszeitungen als der erstrebenswerte Lebensstil vermarktet. Der Sicherheitsaspekt wird aber auf dieser Ebene nie explizit thematisiert. Ebenso wird das Thema Sicherheit in den Bewohnerinterviews nie aus freien Beweggründen berührt. Weder eigene Gewalterfahrungen noch Überfälle auf Verwandte, Freunde oder Bekannte spielen für die Entscheidung zum Umzug in die geschützte Nachbarschaft eine Rolle. Auf direkte Rückfrage sind bisweilen auch ablehnende Äußerungen zu hören:

«Meine Motivation, das ist eine Mischung: meine größeren Töchter leben hier in der Gegend, es gibt mehr Ruhe, mehr Frieden, das Problem der Sicherheit besorgt mich nicht. Ich habe in den letzten Jahren keinerlei Probleme gehabt. Vor 15 Jahren haben sie mir eine Uhr in Corrientes und Uruguay (Straßenkreuzung im Zentrum von Buenos Aires, d. Verf.) geraubt. Ich war im Auto, es war Frühling, deswegen hatte ich die Fensterscheiben runtergekurbelt. Da haben sie mich beklaut. Das war vor 15 Jahren. Was mich ganz schön stört ist, dass sie mich ständig anhalten und mich fragen, wohin ich fahre, wer ich bin, woher ich komme, womit ich mich auf dem Weg vergnüge. Also, diese Sache, welche die persönliche Freiheit einschränkt, die stört mich.» (Guillermo L.)

«Wir haben uns auch Häuser angeschaut, die nicht in einem barrio cerrado lagen. Das Problem der Unsicherheit, das habe ich nicht als etwas Dramatisches angesehen.» (Sergio M.)

\section{Die Mär der Flucht vor Gewalt}

Die Auswertung und Analyse der durchgeführten biographischen Interviews unterstreicht am Fallbeispiel Buenos Aires, dass kein direkter Zusammenhang zwischen der Bedrohung durch Kriminalität und dem Wunsch nach dem Eigenheim in der privaten Nachbarschaft besteht. Inwiefern diese Aussage auch auf andere lateinamerikanischen Metropolen übertragen werden kann, muss durch weitere empirische Untersuchungen geklärt werden. Die Motivation der Bewohner, exemplarisch am Beispiel der größten Urbanisierung im Umland von Buenos Aires, dem Nordelta, durchgeführt, besteht vor allem in einer Verbesserung der individuellen Lebensumstände. Hierbei ist bei den überwiegend jungen Eltern die Bewegungsfreiheit der Kinder in einem geschützten Raum von primärer Bedeutung. Des weiteren wird die großstädtische Lebensweise bewusst abgelehnt. Die Bedrohung durch Gewalttaten ist hingegen auf der Nachfragerseite nur ein untergeordneter Gesichtspunkt, wenngleich sie von den Immobilienfirmen als ein zentrales Verkaufsargument genutzt wird. Der Boom von bewachten Wohnkomplexen im suburbanen Raum von Buenos Aires wird durch das gemein- 
same Marketing von Medien und Immobilienfirmen gefördert und durch eine weitgehend passiv agierende Verwaltung unterstützt. Für das Produkt «Wohnen im suburbanen Raum» gibt es für die Zielgruppen der Mittelschicht und oberen Mittelschicht nur wenig Alternativen zum barrio privado.

\section{Dank}

Die vorliegende Arbeit wurde freundlicherweise unterstützt durch ein Stipendium der Humboldt-RitterPenck-Stiftung der Gesellschaft für Erdkunde zu Berlin.

\section{Literatur}

Amendola, G. (2000): La Ciudad Postmoderna. Magia y Miedo de la Metrópolis Contemporánea. - Madrid: Celeste Ediciones.

BÄHR, J. \& G. MERTINS (1995): Die lateinamerikanische Gross-Stadt. Verstädterungsprozesse und Stadtstrukturen. - = Erträge der Forschung 288, Darmstadt: Wissenschaftliche Buchgesellschaft.

Blakely, E.J. \& M.G. SNyder (1997): Fortress America: gated communities in the United States. - Washington, Cambridge (Massachusetts): Brookings.

Blanco, J.O. (1999): Transporte y espacio urbano en Buenos Aires: Reestructuración de la red de autopistas metropolitanas y cambios en la organización espacial. - In: Actas del I. Encuentro Humboldt, Buenos Aires, 1.-5.11.1999 (Tagungsunterlagen auf CD-ROM).

Borja, J. \& M. CASTells (1997): Local y global. La gestión de las ciudades en la era de la información. Madrid: Santillana de Ediciones - Taurus.

Caldeira, T. (2000): City of Walls. Crime, Segregation and Citizenship in São Paulo. - Berkeley, Los Angeles, London: University of California Press.

Ciccolella, P. (1998): Territorio de consumo. Redefinición del espacio en Buenos Aires en el fin del siglo. - In: Gorenstein, S. \& R. Bustos Cara (Hrsg.): Ciudades y Regiones frente al Avance de la Globalización. - Bahia Blanca: Universidad Nacional del Sur.

Coy, M. (2001): Globalisierung in Brasilien: Raumwirksamkeit und Reaktionen. Beispiele aus städtischen und ländlichen Regionen. - In: Borsdorf, A. \& J. STÖTtER (Hrsg.): Lateinamerika im Umbruch. Geistige Strömungen im Globalisierungsprozess. - = Innsbrukker Geographische Studien 32, Innsbruck: 19-34.

DammerT, L. (2001): Construyendo ciudades inseguras: temor y violencia en Argentina. - In: eure 27, $\mathrm{N}^{\circ} 82$ : 5-20.

Davis, M. (1990): City of Quartz: Excavating the future in Los Angeles. - London: Verso.

Denzin, N.K. (1978): The research act: a theoretical introduction to sociological methods. - 2. Auflage, New York: McGraw-Hill.
Fischer-Rosenthal, W. \& G. Rosenthal (1997): Narrationsanalyse biographischer Selbstpräsentationen. In: Hitzler, R. \& A. Honer (Hrsg.): Sozialwissenschaftliche Hermeneutik. - Opladen: 133-164.

FLICK, U. (2000): Triangulation in der qualitativen Forschung. - In: Flick, U., v. Kardoff, E. \& I. Steinke (Hrsg.): Qualitative Forschung. Ein Handbuch. - Reinbek: 309-318.

Flick, U., v. Kardoff, E. \& I. Steinke (2000): Was ist qualitative Forschung? Einleitung und Überblick. - In: Flick, U., v. KaRdoff, E. \& I. SteInKe (Hrsg.): Qualitative Forschung. Ein Handbuch. - Reinbek: 13-29.

Gilbert, A. (1998): The Latin American City. 2., überarbeitete Version. - London: Latin America Bureau.

Glaser, B.G. \& A.L. Strauss (1998): Grounded Theory. Strategien qualitativer Forschung. - Bern: Huber.

Glasze, G. (2002): Die fragmentierte Stadt. Ursachen und Folgen bewachter Wohnkomplexe im Libanon. Dissertation am Geographischen Institut der Johannes Gutenberg-Universität Mainz (im Druck).

HiernauX-Nicolás, D. (1999): Los frutos amargos de la globalización: Expansión y reestructuración metropolitana de la ciudad de México. - In: eure $25, \mathrm{~N}^{\circ} 76$ : 57-78.

JANOSCHKA, M. (2000): Reich und arm in Buenos Aires. Barrios privados als neue Form der Suburbanisierung. - In: Praxis Geographie 30, $N^{\circ} 12: 60-62$.

JANOSCHKA, M. (2002a): «Stadt der Inseln». Abschottung und Fragmentierung als Zeichen eines neuen Stadtmodells. - In: RaumPlanung 101: 65-70.

JanoschKa, M. (2002b): Wohlstand hinter Mauern. Private Urbanisierungen in Buenos Aires. - Diplomarbeit, Geographisches Institut der Humboldt-Universität zu Berlin, ISR-Forschungsbericht 28, Wien: Verlag der Österreichischen Akademie der Wissenschaften (im Druck).

JANOSCHKA, M. (2002c): Urbanizaciones privadas en Buenos Aires: ¿hacia un nuevo modelo de ciudad latinoamericana? - In: L.F. Cabrales Barajas (Coord.): Latinoamérica: países abiertos, ciudades cerradas. Guadalajara, Paris: Pandora.

LAMNEK, S. (1993): Qualitative Sozialforschung. Band 2: Methoden und Techniken. - Weinheim: Beltz Psychologie Verlags-Union.

Lamnek, S. (1998): Gruppendiskussion. Theorie und Praxis. - Weinheim: Beltz Psychologie Verlags-Union.

Lichtenberger, E. (1998): Stadtgeographie. Bd. 1, Begriffe, Konzepte, Modelle, Prozesse. - Stuttgart, Leipzig: Teubner.

Loos, P. \& B. SCHÄFfer (2001): Das Gruppendiskussionsverfahren. - = Qualitative Sozialforschung, Band 5, Opladen: Leske + Budrich.

Low, S. (2002): Unlocking the Gated Community: Moral Minimalism and Social (Dis)order in Gated Communities in the United States and Mexico. - Vortragsmanuskript, International Conference on «Pri- 
vate Urban Governance», Geographisches Institut, Universität Mainz, 5.-9. Juni 2002.

Marti I Puig, S. (2001): L'Amérique Latine des années 1990: La décennie des opportunités ou celle des chimeres? - In: PréVôt-SchapIRA, M.-F. (Coord.): Métropoles d'Amérique latine: de l'espace public aux espaces privés. - = Cahiers des Amériques Latines 35, Paris: 141-160.

Massey, D. (1999): On Space and the city. - In: Massey, D., Allen, J. \& S. Pile (Hrsg.): City Worlds. - London, New York: 157-175.

MAYRING, P. (1991): Qualitative Inhaltsanalyse. - In: Flick, U. et al. (Hrsg.): Handbuch Qualitative Sozialforschung: Grundlagen, Konzepte, Methoden, Anwendungen. - München: Psychologie Verlags-Union.

MAYRING, P. (1999): Einführung in die qualitative Sozialforschung. Eine Anleitung zu qualitativem Denken. - 4. Auflage, Weinheim: Beltz Psychologie VerlagsUnion.

MAYRING, P. (2000): Qualitative Inhaltsanalyse. Grundlagen und Techniken. - 7. Auflage, Weinheim: Beltz Psychologie Verlags-Union.

MÜller, W. \& R. RoHR-ZäNKER (2001): Amerikanisierung der «Peripherie» in Deutschland. - In: BRAKE, K., Dangschat, J. \& G. Herfert (Hrsg.): Suburbanisierung in Deutschland. Aktuelle Tendenzen. - Opladen: 27-39.

Oehrlein, J. (2002): Ein Leben im Country-Stil. In Argentinien wohnen immer mehr Menschen aus Angst vor Überfällen in geschlossenen Siedlungen. - In: Frankfurter Allgemeine Zeitung, 23.07.2002, Frankfurt.

PrÉvôT-Schapira, M.-F. (2000): Segregación, fragmentación, secesión. Hacia una nueva geografía social en la aglomeración de Buenos Aires. - In: Economía, Sociedad y Territorio 2, №7: 405-431.

RadA, U. (1999): Die Grenzen der Sicherheit. - In: Tageszeitung 5919, 23.08.1999, Berlin: 19.

SchüTZE, F. (1983): Biographieforschung und narratives Interview. - In: Neue Praxis 3: 283-293.

Sennetr, R. (1971): The Uses of Disorder. Personal identity and city life. - London: Lane.

Siegele, L. (1996): Wohlstand hinter Mauern. Aus Angst vor Kriminellen ziehen immer mehr Amerikaner in umzäunte und bewachte Siedlungen. - In: Die Zeit 50, 06.12.1996, Hamburg: 28.

SoJA, E. (2000): Postmetropolis: critical studies of cities and regions. - Oxford, Malden (Massachusetts): Blackwell.

Welch Guerra, M. (2002): Gartentürme des Wohlstands. Buenos Aires: Projektionen einer Wohnhaustypologie. - In: RaumPlanung 101: 71-76.

Wehrheim, J. (1999): Gated Communities: Sicherheit und Separation in den USA. - In: RaumPlanung 87: 248-254.

YANES, L. et al. (1996): Impacto territorial y socioeconómico de la privatización y desregulación de empresas y actividades del transporte en Argentina. In: Memorias del VIII Congreso Latinoamericano de Transporte Público y Urbano, Curritiba: 181-206.

\section{Zusammenfassung: Die Flucht vor Gewalt? Stereo- type und Motivationen beim Andrang auf barrios privados in Buenos Aires}

Entgegen der medialen Berichterstattung zeigen die empirischen Erfahrungen in Buenos Aires, dass die Flucht in bewachte Wohnkomplexe keinen monokausalen Rückzug vor wachsender Gewalt darstellt. Der ausgeprägte Suburbanisierungsdrang konnte in den 1990er Jahren mit der Stabilisierung des Kreditwesens und nach dem Ausbau der Autobahnen von vielen jungen Familien der Mittelschicht verwirklicht werden. Dies stellt auch eine Konsequenz der unzureichenden Lebensbedingungen in zentralen städtischen Vierteln dar. Die verbreitete Umzäunung der neuen Wohnviertel ist dabei eine Strategie von Immobilienfirmen, die private Stadtentwicklung organisieren und sich so höhere Gewinnmargen versprechen. Wenngleich das Marketing der Firmen die Komponente Sicherheit in den Mittelpunkt stellt, spiegelt sich diese Verkaufsstrategie nicht in den Motivationen der Bewohner wider. Diese drehen sich um Aspekte, die aus den Suburbanisierungsprozessen in Westeuropa bekannt sind.

\section{Summary: Escape from Criminality? Rush for gated communities in Buenos Aires - stereotypes and motivation}

Contrary to media reports, empirical field work in Buenos Aires shows that the flight of people away from the city centres towards gated communities is not only a result of growing crime rates. The stabilization of the banking and credit sector, improvement of metropolitan motorway networks, and deteriorating living conditions in central areas also contributed noticeably to the strong suburbanisation trend of young middle class families in the 1990's. Although the aspect of security plays a central role in marketing these suburban residential areas, it does not play a significant role in the decision-making process of potential residents. Rather, the reasons mentioned for moving to the suburbs correspond to those articulated by young families in Western Europe. Thus, the enclosure of newly developed residential areas in the suburbs may be interpreted as a strategy by private property developers to increase the profitability of new projects.

\section{Résumé: La fuite face à la criminalité? Stéréotypes et motivations de l'évasion vers les quartiers enclos à Buenos Aires}

Contrairement aux reportages médiatiques, les expériences empiriques relatives à Buenos Aires démontrent que la fuite des habitants vers les quartiers enclos ne se réduit pas à une explication mono-causale de la pro- 
tection contre la violence. Au cours des années 1990 , l'attrait de la suburbanisation a pu se concrétiser pour de nombreux jeunes ménages des classes moyennes, à la faveur de la stabilisation du crédit et consécutivement à l'aménagement du réseau autoroutier. Ce phénomène s'explique aussi par les mauvaises conditions de vie dans les quartiers urbains centraux. La pratique de la clôture des nouveaux quartiers d'habitation relève du déploiement d'une stratégie propre aux firmes immobilières qui organisent un développement urbain privé en vue d'une augmentation de leurs marges bénéficiaires. Quoique le marketing des firmes place la notion de sécurité au centre des préoccupations, cette stratégie de vente ne reflète aucunement les motivation des habitants. Celles-ci tournent plutôt autour d'aspects courants dans les processus de suburbanisation en Europe occidentale.

\section{Didaktische Hinweise}

- Wie entstehen barrios privados in Buenos Aires?

- Welche Gründe bewegten Private zum Einzug in ein geschütztes Wohngebiet? Wie lassen sich die Neubewohner biographisch typologisieren?
- Welchen Stellenwert hat das Motiv «Angst» in der Vermarktung von geschlossenen Siedlungen und bei den Umzügern?

- Worin unterscheidet sich JANOSCHKAS Untersuchungsmethode von jener anderer Autoren?

Dipl.-Geogr. Michael Janoschka, Teltower Damm 266, D-14167 Berlin.

e-mail:m.janoschka@berlin.de

Manuskripteingang/received/manuscrit entré le 30.8.2002

Annahme zum Druck/accepted for publication/accepté pour l'impression: 4.12 .2002 